\title{
Short-range plasma model for intermediate spectral statistics
}

\author{
Eugène Bogomolny ${ }^{1}$, Ulrich Gerland ${ }^{2}$ and Charles Schmit ${ }^{1}$ \\ ${ }^{1}$ Laboratoire de Physique Théorique et Modèles Statistiques*, Univ. Paris-Sud, 91405 Orsay \\ Cedex, France \\ ${ }^{2}$ Phys. Dep., UCSD, La Jolla, CA 92093-0319, USA
}

(October 26, 2018)

PACS numbers: 05.45.-a, 03.65.Sq, 05.40.-a

\begin{abstract}
We propose a plasma model for spectral statistics displaying level repulsion without long-range spectral rigidity, i.e. statistics intermediate between random matrix and Poisson statistics similar to the ones found numerically at the critical point of the Anderson metal-insulator transition in disordered systems and in certain dynamical systems. The model emerges from Dysons one-dimensional gas corresponding to the eigenvalue distribution of the classical random matrix ensembles by restricting the logarithmic pair interaction to a finite number $k$ of nearest neighbors. We calculate analytically the spacing distributions and the two-level statistics. In particular we show that the number variance has the asymptotic form $\Sigma^{2}(L) \sim \chi L$ for large $L$ and the nearest-neighbor distribution decreases exponentially when $s \rightarrow \infty$, $P(s) \sim \exp (-\Lambda s)$ with $\Lambda=1 / \chi=k \beta+1$, where $\beta$ is the inverse temperature of the gas $(\beta=1,2$ and 4 for the orthogonal, unitary and symplectic symmetry class respectively). In the simplest case of $k=\beta=1$, the model leads to the so-called Semi-Poisson statistics characterized by particular simple correlation functions e.g. $P(s)=4 s \exp (-2 s)$. Furthermore we investigate the spectral statistics of several pseudointegrable quantum billiards numerically and compare them to the Semi-Poisson statistics.
\end{abstract}

*Unité de Recherche de l'Université Paris XI et du CNRS (UMR 8626) 


\section{INTRODUCTION}

Since the advent of random matrix theory there has been considerable interest in the statistical analysis of spectra [1] 4 ]. Two diametrically opposed statistical distributions have been found to be of universal relevance: the Poisson distribution, i.e. completely uncorrelated levels, and the Wigner-Dyson distributions of random matrix theory. The prominent features of these distributions are conveniently characterized with the help of spectral observables such as the nearest-neighbor spacing distribution $P(s)$ and the number variance $\Sigma^{2}(L)$ (the variance of the number of levels in an energy interval of length $L$ in the unfolded spectrum). The former stresses the correlations on a short scale, while the latter measures the stiffness of the spectrum, i.e. long-range spectral correlations. For the standard random matrix ensembles (orthogonal, unitary and symplectic symmetry labeled by $\beta=1,2$ and 4 respectively) the spacing distribution is approximately given by the Wigner surmise and the number variance increases only logarithmically with large $L$,

$$
\begin{aligned}
P(s) & =a_{\beta} s^{\beta} \exp \left(-c_{\beta} s^{2}\right) \\
\Sigma^{2}(L) & \sim \frac{2}{\beta \pi^{2}} \log (L) \quad(L \rightarrow \infty)
\end{aligned}
$$

( $a_{\beta}$ and $c_{\beta}$ are determined by normalization and by requiring the mean level spacing to be one). Thus the Wigner-Dyson distributions are marked by level repulsion and long-range spectral rigidity. In contrast, for the Poisson distribution one has neither level repulsion nor spectral rigidity,

$$
\begin{aligned}
P(s) & =\exp (-s) \\
\Sigma^{2}(L) & =L .
\end{aligned}
$$

In the present article we devise a discrete set of statistical distributions with properties intermediate between those of the Poisson and Wigner-Dyson distributions, namely level repulsion

$$
P(s) \sim s^{\beta} \quad(s \rightarrow 0)
$$

paired with an exponential decay of the nearest-neighbor spacing distribution,

$$
P(s) \sim \exp (-\Lambda s) \quad(s \rightarrow \infty)
$$

and a linear asymptotic of the number variance,

$$
\Sigma^{2}(L) \sim \chi L \quad(L \rightarrow \infty)
$$

Our distributions arise as the Gibbs distribution of a one-dimensional gas of interacting

classical particles. It was noted already by Dyson that the distribution of eigenvalues $E_{j}$ of random matrices is equivalent to the distribution of particles in a one-dimensional gas with inverse temperature $\beta$ and the repulsive two-body interaction potential $V(x)=-\log |x|$, since the joint probability distribution can be written in the form

$$
P_{N \beta}\left(E_{1}, \ldots, E_{N}\right)=Z_{N}^{-1} \mathrm{e}^{-\beta W\left(E_{1}, \ldots, E_{N}\right)},
$$


where the normalization constant $Z_{N}$ is the canonical partition function of the gas and

$$
W\left(E_{1}, \ldots, E_{N}\right)=\sum_{i} U\left(E_{i}\right)+\sum_{i<j} V\left(\left|E_{i}-E_{j}\right|\right)
$$

is the total potential energy. The one-body potential $U$ serves to confine the $E_{j}$ to a finite stretch of the $E$-axis, e.g. $U(E) \propto E^{2}$ for the Gaussian ensembles. It determines the mean particle (level) density, but it is irrelevant for the statistical correlations on the scale of the mean level spacing (random matrix universality) as long as $U$ is steep enough to actually confine the levels [5].

Our plasma model of intermediate spectral statistics is defined by the same eqs. (6) and (7), except that we restrict the second sum on the right hand side of eq. (7) to a finite number $k$ of nearest neighbors. It is this screening of the Coulomb interaction between the levels, which is the essential ingredient leading to the properties eqs. (3)-(5).

Our first motivation stems from the physics of disordered electronic systems. A 3dimensional disordered sample undergoes a phase transition between an insulating and a metallic phase as a function of the disorder strength (Anderson metal-insulator transition). In the insulating phase the electron eigenfunctions are localized and since non-overlapping eigenfunctions are uncorrelated, the eigenenergies are Poisson distributed. In contrast, in the metallic phase the eigenfunctions extend homogeneously over the whole sample and overlap strongly which leads to a Wigner-Dyson distribution of the energy levels. Exactly at the transition point the electron eigenfunctions are extended, but strongly inhomogeneous (multifractal). This leads to intermediate spectral statistics, which are believed to be universal, i.e. independent of the microscopic details of the disordered system [6].

Fyodorov and Mirlin [7] calculated the overlap of two critical eigenstates with slightly different energy and found that the overlap is one if the energy separation, $s$, between the eigenfunctions is of the order of mean level spacing, while for larger $s$ it decays as a certain power of $s$. In contrast, for Wigner-Dyson statistics the overlap is one for all values of $s$. Hence one may conclude that at the critical point of the Anderson metal-insulator transition only eigenvalues which are separated by at most a few level spacings interact strongly.

A second motivation which led us to consider the short-range plasma model is the following. In the context of quantum chaos the statistical analysis of spectra plays a major role. In the semiclassical limit the quantum energy spectra of systems with integrable classical dynamics generally display Poisson statistics [8], while the Wigner-Dyson distribution is associated with fully chaotic classical dynamics [9]. But there are systems which are neither integrable nor chaotic. In [10] we examined numerically a few such systems and found that their spectral statistics exhibit all properties (3) - (5) typical for intermediate statistics. In particular, the simple expression

$$
P(s)=4 s e^{-2 s}
$$

is an excellent fit to the spacing distribution of a subclass of systems considered. We further noticed that the short-range plasma model with nearest-neighbor interaction $(k=1)$ leads precisely to this distribution (and we checked that other correlation functions are also well described by this model).

Another interesting class of plasma models with screened Coulomb interaction is the Gaudin model [11,12] defined by eqs. (6) and ([7) with the two-body interaction potential 


$$
V(x)=-\frac{1}{2} \log \frac{x^{2}}{a^{2}+x^{2}} .
$$

When $a \rightarrow \infty$ it reduces to the random matrix models, while in the limit $a \rightarrow 0$ it yields Poisson statistics. For $\beta=2$ the model is solvable and all correlation functions can be written in a closed form [11, 12]. They obey all three conditions (3) - (5) characteristic for intermediate statistics with the following constants (for $\beta=2$ )

$$
\chi=\frac{1}{\alpha}\left(1-e^{-\alpha}\right)
$$

and

$$
\Lambda=\frac{1}{\alpha} \int_{0}^{e^{\alpha}-1} \frac{\ln (1+t)}{t} d t .
$$

Here $\alpha=2 \pi a \rho$ and $\rho$ is the mean density of the levels.

In general, in any plasma model with short-range interaction the nearest-neighbor spacing distribution will decay exponentially at large distances. It is the otherwise unusual property of long-range interaction in the standard random matrix models (which manifests itself in the summation over all pairs of particles in eq. (7)) that is responsible for the $\exp \left(-c s^{2}\right)$ decay of $P(s)$ at large $s$.

The main drawback of the Gaudin model is that no analytical solution is known for $\beta \neq 2$. In contrast, our model can be solved for arbitrary $\beta$ (and arbitrary potential).

We stress that our one-dimensional gas model is meant as a toy model which deserves interest, because it provides a natural discrete interpolation between Poisson and WignerDyson statistics, it is analytically solvable, and it is physically motivated. It does not pretend to furnish an exact description of the critical statistics of a physical model (e.g. the Anderson model in the MIT point). Despite significant theoretical progress (see [7], [17], [24]- [26] and references therein), the precise form of the critical distribution is not yet known though there exist arguments ( 24]- 26]) that the main difference between the standard random matrix ensembles and the intermediate statistics is in the form of the correlation kernel, $K(x, y)$, which determines the correlation functions in the random matrix theory [2]. In the standard ensembles

$$
K(x, y)=\frac{\sin \pi(x-y)}{\pi(x-y)} .
$$

For intermediate statistics it has been argue that

$$
K(x, y) \approx \frac{a \sin \pi(x-y)}{\pi \sinh a(x-y)},
$$

where $a$ is a parameter. When $a \rightarrow 0$ the standard random ensembles are recoved. Non-zero value of $a$ characterizes the intermediate statistics. The detailed discussion of this type of critical behavior is beyond the scope of this paper.

The paper is organized as follows. In the next section we present the calculation of the spacing distributions and the number variance in the one-dimensional plasma, first for $k=1$, then for $k=2$, and finally for arbitrary $k$. We compare the resulting distributions with our numerical results for the pseudointegrable billiards in section III. Finally, in the discussion of section IV we compare the short-range plasma model to other existing interpolations between Poisson and Wigner-Dyson statistics. 


\section{THE MODEL}

Instead of a linear one-dimensional gas as in eqs. (6) and (7), where the levels are confined by a one-body potential $U(E)$, we choose a circular geometry. This is convenient, since on a circle the levels are automatically confined and it is unnecessary to introduce an external potential. The mean level density is then constant and unfolding is trivial. At the same time the correlations in the unfolded level sequence are the same as in the linear geometry (in the limit of a large number of levels), just as the Gaussian ensembles of random matrix theory are locally equivalent to Dyson's circular ensembles. The method of calculation that we apply in this section goes back to Gürsey 13 for the case of nearest-neighbor interaction, and to van Hove [14 for the general case (see also ref. [15]).

We consider $N$ particles (representing energy levels) on a circle of circumference $L$. We denote the positive spacings between neighboring particles by $\xi_{j}$ with $j=1 \ldots N$ (see Fig. 1). Hence,

$$
\sum_{j=1}^{N} \xi_{j}=L .
$$

For convenience we use a periodic index, i.e. $\xi_{j+N}:=\xi_{j}$. We introduce an interaction between the particles via a repulsive two-body potential $V(\xi)$ (eventually we will choose $V(\xi)=-\log |\xi|$ as in random matrix theory), but we let each particle interact only with its $k$ nearest neighbors to the left and to the right $(k=1,2,3, \ldots)$.

The canonical partition function of this one-dimensional gas is

$$
\begin{aligned}
Z_{N}(L, \beta)= & \int_{0}^{\infty} \mathrm{d} \xi_{1} \ldots \int_{0}^{\infty} \mathrm{d} \xi_{N} \delta\left(L-\sum_{i=1}^{N} \xi_{i}\right) \times \\
& \exp \left(-\beta \sum_{j=1}^{N} W\left(\xi_{j}, \ldots, \xi_{j+k-1}\right)\right),
\end{aligned}
$$

where

$$
\begin{aligned}
W\left(\xi_{j}, \ldots, \xi_{j+k-1}\right)= & V\left(\xi_{j}\right)+V\left(\xi_{j}+\xi_{j+1}\right)+ \\
& \ldots+V\left(\xi_{j}+\ldots+\xi_{j+k-1}\right) .
\end{aligned}
$$

$W\left(\xi_{j}, \ldots, \xi_{j+k-1}\right)$ includes the interaction energy of the particle which is located at the left of $\xi_{j}$ with its $k$ nearest right neighbors only to avoid double counting. Since for the application to spectral statistics $\beta$ is not a free variable, but takes only the values 1,2 , and 4 , we suppress the $\beta$-dependence of the partition function in the following. For brevity of notation we define

$$
f(\xi):=\exp (-\beta V(\xi))
$$

which for the case of $V(\xi)=\ln |\xi|$ becomes

$$
f(\xi)=|\xi|^{\beta} .
$$

The joint probability distribution of $n$ consecutive spacings then takes the form 


$$
\begin{gathered}
p\left(\xi_{1}, \ldots, \xi_{n}\right)=\frac{1}{Z_{N}(L)} \int_{0}^{\infty} \mathrm{d} \xi_{n+1} \ldots \int_{0}^{\infty} \mathrm{d} \xi_{N} \delta\left(L-\sum_{i=1}^{N} \xi_{i}\right) \\
\times \prod_{j=1}^{N} f\left(\xi_{j}\right) f\left(\xi_{j}+\xi_{j+1}\right) \cdots f\left(\xi_{j}+\ldots+\xi_{j+k-1}\right) .
\end{gathered}
$$

In the following the variable $s$ denotes distances measured in units of the mean spacing $\Delta=L / N$. Our aim is to calculate correlation functions such as the $n$-th nearest-neighbor spacing distribution $P(n, s)$ (the distribution of the distance $s$ between two particles that have $n$ particles between them) and the two-point correlation function $R_{2}(s)$ (the probability of finding any two particles at distance $s$ ). The latter is related to the former by summation over $n$,

$$
R_{2}(s)=\sum_{n=0}^{\infty} P(n, s) .
$$

Using eq. (18) the $n$-th nearest-neighbor spacing distribution can be expressed as

$$
\begin{gathered}
P(n, s)=\int_{0}^{\infty} \mathrm{d} \xi_{1} \ldots \int_{0}^{\infty} \mathrm{d} \xi_{n+1} \delta\left(s-\frac{1}{\Delta} \sum_{i=1}^{n+1} \xi_{i}\right) \times \\
p\left(\xi_{1}, \ldots, \xi_{n+1}\right) .
\end{gathered}
$$

The number variance can be obtained from the two-point correlation function with help of the relation

$$
\Sigma^{2}(L)=L-2 \int_{0}^{L} \mathrm{~d} s(L-s)\left(1-R_{2}(s)\right) .
$$

A convenient way to calculate the asymptotic behavior of the number variance for large $L$ is to consider the asymptotic expansion of the Laplace transform of the two-point correlation function,

$$
g_{2}(t)=\int_{0}^{\infty} \mathrm{d} s R_{2}(s) e^{-t s}
$$

for small $t$. If

$$
g_{2}(t)=\frac{1}{t}+\alpha_{0}+\alpha_{1} t+O\left(t^{2}\right) \quad(t \rightarrow 0)
$$

then we get from eq. (21)

$$
\Sigma^{2}(L)=\chi L+C+O\left(L^{-1}\right) \quad(L \rightarrow \infty),
$$

where $\chi=1+2 \alpha_{0}$ and $C=2 \alpha_{1}$ (for the determination of the constant term we have assumed that $R_{2}(s)$ falls off faster than $1 / s^{2}$, which is true for the cases considered in this article). 


\section{A. Nearest-neighbor interaction $(k=1)$}

We begin with the simplest case, where the interaction is restricted to nearest neighbors, so that the expression for the partition function eq. (14) simplifies to

$$
Z_{N}(L)=\int_{0}^{\infty} \mathrm{d} \xi_{1} \ldots \int_{0}^{\infty} \mathrm{d} \xi_{N} \delta\left(L-\sum_{i=1}^{N} \xi_{i}\right) \prod_{j=1}^{N} f\left(\xi_{j}\right) .
$$

By Laplace transformation with respect to $L$ we obtain

$$
\begin{aligned}
g_{N}(t) & :=\int_{0}^{\infty} \mathrm{d} L Z_{N}(L) e^{-t L} \\
& =\left(\int_{0}^{\infty} \mathrm{d} x f(x) e^{-t x}\right)^{N}=:[g(t)]^{N} .
\end{aligned}
$$

The large $N$ limit of the partition function can now be calculated by performing the Laplace inversion in saddle point approximation. We have

$$
\begin{aligned}
Z_{N}(L) & =\frac{1}{2 \pi i} \int_{c-i \infty}^{c+i \infty} \mathrm{d} t g_{N}(t) e^{L t} \\
& =\frac{1}{2 \pi i} \int_{c-i \infty}^{c+i \infty} \mathrm{d} t e^{N(t \Delta+\log g(t))} \\
& \sim[g(c)]^{N} e^{L c}
\end{aligned}
$$

where $\Delta=L / N$ is the mean level spacing and $c$ is determined from the saddle point equation

$$
\Delta+\frac{g^{\prime}(c)}{g(c)}=0
$$

The expression for the joint probability distribution of $n$ consecutive spacings eq. (18) reduces in the present case to

$$
p\left(\xi_{1}, \ldots, \xi_{n}\right)=\frac{Z_{N-n}\left(L-\sum_{i=1}^{n} \xi_{i}\right)}{Z_{N}(L)} \prod_{j=1}^{n} f\left(\xi_{j}\right) .
$$

Using eq. (27) and assuming $n \ll N$ we obtain

$$
p\left(\xi_{1}, \ldots, \xi_{n}\right)=\prod_{j=1}^{n} \frac{1}{g(c)} e^{-c \xi_{j}} f\left(\xi_{j}\right) .
$$

Note that the factor $g(c)^{-1}$ assures the proper normalization,

$$
\int_{0}^{\infty} \mathrm{d} \xi_{n} p\left(\xi_{1}, \ldots, \xi_{n}\right)=p\left(\xi_{1}, \ldots, \xi_{n-1}\right) .
$$


With help of eqs. (20) and (29) we find for the Laplace transformation of the $n$-th nearestneighbor spacing distribution

$$
g(n, t):=\int_{0}^{\infty} \mathrm{d} s e^{-t s} P(n, s)=\left[\frac{g(c+t / \Delta)}{g(c)}\right]^{n+1}
$$

Using the relation (19) we calculate the Laplace transform of the two-point correlation function, eq. (22),

$$
g_{2}(t)=\frac{g(c+t / \Delta)}{g(c)-g(c+t / \Delta)} .
$$

The small- $t$ asymptotic behavior is

$$
\begin{aligned}
g_{2}(t)= & \frac{1}{t}-1-\frac{1}{2 \Delta} \frac{g^{\prime \prime}(c)}{g^{\prime}(c)}+ \\
& +\frac{3 g^{\prime \prime}(c)^{2}-2 g^{\prime}(c) g^{(3)}(c)}{12 g^{\prime}(c)^{2}} \frac{t}{\Delta^{2}}+O\left(t^{2}\right)
\end{aligned}
$$

where we have employed the saddle point equation (28).

At this point we specialize to the random matrix interaction potential, i.e. we substitute eq. (17). Its Laplace transform reads

$$
g(t)=\frac{\Gamma(\beta+1)}{t^{\beta+1}}
$$

and the saddle point equation (28) yields

$$
c=\frac{\beta+1}{\Delta}
$$

By Laplace inversion of eq. (30) we then obtain the $n$-th nearest-neighbor spacing distribution

$$
P(n, s)=\frac{(\beta+1)^{(n+1)(\beta+1)}}{\Gamma((\beta+1)(n+1))} s^{\beta+n(\beta+1)} \mathrm{e}^{-(\beta+1) s}
$$

In particular, the nearest-neighbor spacing distribution for $\beta=1,2,4$ is

$$
\begin{array}{ll}
P(s)=4 s \mathrm{e}^{-2 s} & (\beta=1), \\
P(s)=\frac{27}{2} s^{2} \mathrm{e}^{-3 s} & (\beta=2), \\
P(s)=\frac{3125}{24} s^{4} \mathrm{e}^{-5 s} & (\beta=4) .
\end{array}
$$

The Laplace transform of the two-point correlation function eq. (31) becomes

$$
g_{2}(t)=\frac{1}{\left(1+\frac{t}{\beta+1}\right)^{\beta+1}-1},
$$


from which $R_{2}(s)$ can be computed by Laplace inversion.

$$
\begin{aligned}
& R_{2}(s)=1-\exp (-4 s) \quad(\beta=1) \\
& R_{2}(s)=1-(\cos (3 \sqrt{3} s / 2)+ \\
& \sqrt{3} \sin (3 \sqrt{3} s / 2)) e^{-9 s / 2} \quad(\beta=2) \\
& R_{2}(s)=e^{-5 s} \sum_{k=0}^{4} \exp \left(5 s e^{2 \pi i k / 5}+2 \pi i k / 5\right) \quad(\beta=4)
\end{aligned}
$$

With help of eqs. (23) and (24) and the small $t$ expansion of eq. (37) we find the large $L$ behavior of the number variance $\Sigma^{2}(L) \sim \chi L+C$ with

$$
\chi=\frac{1}{\beta+1}, \quad C=\frac{\beta(\beta+2)}{6(1+\beta)^{2}} .
$$

The spacing distribution and the two-point correlation function of the simplest model with $k=\beta=1$ are very close to the corresponding distributions of a number of different dynamical systems, whose spectral statistics can be calculated only numerically (see sects. III and [V). In lack of a deeper understanding of the spectral statistics of these systems, the plasma model is valuable in that it provides simple fitting distributions to these numerical results. We propose to call the statistics derived from the plasma model with $k=\beta=1$ the Semi-Poisson statistics. This name was originally motivated from the fact that if one takes an ordered Poisson distributed sequence $\left\{x_{n}\right\}$, the nearest-neighbor spacing distribution of the new sequence $\left\{y_{n}\right\}$ with $y_{n}=\left(x_{n}+x_{n+1}\right) / 2$ coincides with eq. (8) (of course the other correlation functions of the sequence $\left\{y_{n}\right\}$ are different from those of the plasma model).

It is interesting to note that again starting from the sequence $\left\{x_{n}\right\}$ and dropping every second level one obtains a new sequence with precisely the same statistical distribution as the plasma model with $k=\beta=1$ [16]. More generally, retaining only every $(p+1)$-th level

of the sequence $\left\{x_{n}\right\}$ leads to the same statistical distribution as the plasma model with $k=1$ and $\beta=p$ [16].

\section{B. Interaction between nearest and next-to-nearest neighbors $(k=2)$}

Next we consider the one-dimensional gas, where the interaction is restricted to nearest and next-to-nearest neighbors. In this case the partition function takes the form

$$
\begin{gathered}
Z_{N}(L)=\int_{0}^{\infty} \mathrm{d} \xi_{1} \ldots \int_{0}^{\infty} \mathrm{d} \xi_{N} \delta\left(L-\sum_{i=1}^{N} \xi_{i}\right) \times \\
\prod_{j=1}^{N} f\left(\xi_{j}\right) f\left(\xi_{j}+\xi_{j+1}\right) .
\end{gathered}
$$

As in the previous section we first compute the Laplace transform of the partition function,

$$
\begin{aligned}
g_{N}(t) & :=\int_{0}^{\infty} \mathrm{d} L Z_{N}(L) e^{-t L} \\
& =\int_{0}^{\infty} \mathrm{d} \xi_{1} \ldots \int_{0}^{\infty} \mathrm{d} \xi_{N} \prod_{j=1}^{N} e^{-t \xi_{j}} f\left(\xi_{j}\right) f\left(\xi_{j}+\xi_{j+1}\right) .
\end{aligned}
$$


To evaluate this integral we introduce the transfer operator [14]

$$
K\left(\xi, \xi^{\prime}\right)=\sqrt{f(\xi)} e^{-t \xi / 2} f\left(\xi+\xi^{\prime}\right) \sqrt{f\left(\xi^{\prime}\right)} e^{-t \xi^{\prime} / 2}
$$

so that $g_{N}(t)$ can be rewritten as

$$
\begin{aligned}
g_{N}(t) & =\operatorname{tr} K^{N} \\
\equiv & \int_{0}^{\infty} \mathrm{d} \xi_{1} \ldots \int_{0}^{\infty} \mathrm{d} \xi_{N} K\left(\xi_{1}, \xi_{2}\right) K\left(\xi_{2}, \xi_{3}\right) \ldots \\
& \ldots K\left(\xi_{N-1}, \xi_{N}\right) K\left(\xi_{N}, \xi_{1}\right) .
\end{aligned}
$$

The operator $K\left(\xi, \xi^{\prime}\right)$ is real symmetric and therefore admits the eigenbasis expansion

$$
K\left(\xi, \xi^{\prime}\right)=\sum_{j} \lambda_{j} \phi_{j}(\xi) \phi_{j}\left(\xi^{\prime}\right)
$$

with real eigenvalues $\lambda_{j}$ and eigenfunctions $\phi_{j}(\xi)$,

$$
\int_{0}^{\infty} \mathrm{d} \xi^{\prime} K\left(\xi, \xi^{\prime}\right) \phi_{j}\left(\xi^{\prime}\right)=\lambda_{j} \phi_{j}(\xi) \text {. }
$$

The eigenfunctions are normalized according to

$$
\int_{0}^{\infty} \mathrm{d} \xi \phi_{j}(\xi) \phi_{j^{\prime}}(\xi)=\delta_{j, j^{\prime}}
$$

and we choose the ordering of the eigenvalues by decreasing magnitude $\left(\lambda_{0}>\lambda_{1}>\ldots\right)$. Consequently, in the large $N$ limit the Laplace transform of the partition function, eq. (39), reduces to

$$
g_{N}(t)=\left[\lambda_{0}(t)\right]^{N}
$$

where we have explicitly indicated the $t$-dependence of the eigenvalue. Again we perform the Laplace inversion in saddle point approximation, which results in $t$ being fixed to $t=c$,

$$
Z_{N}(L) \sim\left[\lambda_{0}(c)\right]^{N} e^{L c}
$$

where $c$ is determined from the saddle point equation

$$
\Delta+\frac{\lambda_{0}^{\prime}(c)}{\lambda_{0}(c)}=0
$$

Next we calculate the joint probability distribution of $n$ consecutive spacings, eq. (18). It turns out to be convenient to write the eigenfunctions in the form

$$
\phi(t ; \xi)=\sqrt{f(\xi)} e^{-t \xi / 2} \psi(t ; \xi)
$$


(again the dependence on $t$ is explicitly indicated). We express the Laplace transform of the integral on the right hand side of eq. (18) in terms of the transfer operator $K$, use the eigenfunction expansion eq. (40), perform the Laplace inversion in saddle point approximation (the saddle point equation is identical with eq. (44)), and arrive at

$$
\begin{aligned}
p\left(\xi_{1}, \ldots, \xi_{n}\right)= & \frac{1}{\lambda_{0}(c)^{n-1}} \psi_{0}\left(c ; \xi_{1}\right) \psi_{0}\left(c ; \xi_{n}\right) e^{-c \sum_{i=1}^{n} \xi_{i}} \\
& f\left(\xi_{n}\right) \prod_{j=1}^{n-1} f\left(\xi_{j}\right) f\left(\xi_{j}+\xi_{j+1}\right) .
\end{aligned}
$$

With the help of eq. (46) we can now calculate all correlation functions. The simplest is the nearest-neighbor spacing distribution

$$
P(s)=\Delta\left[\phi_{0}(c ; s \Delta)\right]^{2} .
$$

To calculate the asymptotic behavior of the number variance we first take the Laplace transform of the $n$-th nearest-neighbor spacing distribution eq. (20) which yields

$$
\begin{aligned}
g(n, t)= & \sum_{j}\left(\frac{\lambda_{j}(c+t / \Delta)}{\lambda_{0}(c)}\right)^{n} \times \\
& {\left[\int_{0}^{\infty} \mathrm{d} \xi \phi_{0}(c ; \xi) \phi_{j}(c+t / \Delta ; \xi) e^{-t \xi / 2 \Delta}\right]^{2} . }
\end{aligned}
$$

Hence, by eq. (19), the Laplace transform of the two-level correlation function takes the form

$$
\begin{aligned}
g_{2}(t)= & \sum_{j} \frac{\lambda_{0}(c)}{\lambda_{0}(c)-\lambda_{j}(c+t / \Delta)} \times \\
& {\left[\int_{0}^{\infty} \mathrm{d} \xi \phi_{0}(c ; \xi) \phi_{j}(c+t / \Delta ; \xi) e^{-t \xi / 2 \Delta}\right]^{2} . }
\end{aligned}
$$

For small $t$ this becomes

$$
g_{2}(t)=\frac{1}{t}-\frac{1}{2 \Delta} \frac{\lambda_{0}^{\prime \prime}(c)}{\lambda_{0}^{\prime}(c)}-1+O(t)
$$

where we have used the saddle point equation (44) and

$$
\frac{1}{\Delta} \int_{0}^{\infty} \mathrm{d} \xi \xi \phi_{0}(c, \xi)^{2}=1
$$

which follows from eq. (47) and the definition of the mean level spacing $\Delta$ (implying $\left.\int \mathrm{d} s s P(s)=1\right)$.

We now specialize to the random matrix interaction eq. (17) and restrict ourselves to the case of $\beta$ integer. The integral equation (41) with the eigenfunctions in the form of eq. (45) then reads 


$$
\int_{0}^{\infty} \mathrm{d} \xi^{\prime} e^{-t \xi^{\prime}} \xi^{\prime \beta}\left(\xi^{\prime}+\xi\right)^{\beta} \psi_{j}\left(t ; \xi^{\prime}\right)=\lambda_{j}(t) \psi_{j}(t ; \xi) .
$$

It is clear from eq. (51) that the $t$ dependence factorizes and

$$
\begin{aligned}
\lambda_{j}(t) & =t^{-2 \beta-1} \lambda_{j}(1), \\
\psi_{j}(t ; \xi) & =t^{(\beta+1) / 2} \psi(1 ; t \xi) .
\end{aligned}
$$

This permits us to determine $c$ from the saddle point equation (44), which yields

$$
c=\frac{2 \beta+1}{\Delta} \text {. }
$$

Using eqs. (23), (24), and (50) we then find the asymptotic behavior of the number variance,

$$
\Sigma^{2}(L) \sim \chi L, \quad \chi=\frac{1}{2 \beta+1} .
$$

In order to determine the spacing distributions we actually need to solve the integral equation (51). It is straightforward to see that it has $\beta+1$ solutions each being a polynomial of degree $\beta$,

$$
\psi(t ; \xi)=\sum_{j=0}^{\beta} a_{j} \xi^{j}
$$

The coefficients $a_{j}$ are easily obtained by substituting eq. (54) into eq. (51). The spacing distributions then follow from eq. (46) and eq. (20). Table II shows the explicit expression for the nearest and next-to-nearest-neighbor spacing distribution for $\beta=1,2$, and 4 .

\section{General case}

Finally we extend the calculation of the previous section to an interaction between an arbitrary number $k$ of neighboring particles. In this general case the Laplace transform of the partition function (14) takes the form

$$
\begin{aligned}
g_{N}(t)= & \int_{0}^{\infty} \mathrm{d} \xi_{1} \ldots \int_{0}^{\infty} \mathrm{d} \xi_{N} \prod_{j=1}^{N} e^{-t \xi_{j}} \times \\
& f\left(\xi_{j}\right) f\left(\xi_{j}+\xi_{j+1}\right) \cdots f\left(\xi_{j}+\ldots+\xi_{j+k-1}\right) .
\end{aligned}
$$

Again we seek to express $g_{N}(t)$ as the trace of the $N$-th power of a transfer operator. To this end we define 14

$$
\begin{aligned}
& K\left(\mathbf{x}, \mathbf{x}^{\prime}\right)=\delta\left(x_{2}-x_{1}^{\prime}\right) \delta\left(x_{3}-x_{2}^{\prime}\right) \cdots \delta\left(x_{k-1}-x_{k-2}^{\prime}\right) \times \\
& e^{-t x_{1} / 2} \sqrt{f\left(x_{1}\right)} \sqrt{f\left(x_{1}+x_{2}\right)} \cdots \sqrt{f\left(x_{1}+\ldots+x_{k-1}\right)} \times \\
& f\left(x_{1}+\ldots+x_{k-1}+x_{k-1}^{\prime}\right) \sqrt{f\left(x_{1}^{\prime}+\ldots+x_{k-1}^{\prime}\right)} \cdots \times \\
& \sqrt{f\left(x_{k-2}^{\prime}+x_{k-1}^{\prime}\right)} \sqrt{f\left(x_{k-1}^{\prime}\right)} e^{-t x_{k-1}^{\prime} / 2},
\end{aligned}
$$


where $\mathbf{x}=\left(x_{1}, \ldots, x_{k-1}\right)$ and $\mathbf{x}^{\prime}$ is defined likewise. One then easily verifies that

$$
\begin{aligned}
& g_{N}(t)=\operatorname{tr} K^{N} \\
& \equiv \int \mathrm{d} \mathbf{x}_{1} \ldots \int \mathrm{d} \mathbf{x}_{N} K\left(\mathbf{x}_{1}, \mathbf{x}_{2}\right) \times \\
& K\left(\mathbf{x}_{2}, \mathbf{x}_{3}\right) \ldots K\left(\mathbf{x}_{N}, \mathbf{x}_{1}\right) .
\end{aligned}
$$

It is clear from the definition (56) that the transfer operator obeys the symmetry relation

$$
K\left(\mathbf{x}, \mathbf{x}^{\prime}\right)=K\left(\mathbf{x}^{\prime T}, \mathbf{x}^{T}\right)
$$

where we use the notation $\mathbf{x}^{T}=\left(x_{k-1}, \ldots, x_{1}\right)$. The presence of this symmetry permits the eigenbasis expansion

$$
K\left(\mathbf{x}, \mathbf{x}^{\prime}\right)=\sum_{j} \lambda_{j} \phi_{j}(\mathbf{x}) \phi_{j}\left(\mathbf{x}^{\prime T}\right)
$$

with the eigenvalues $\lambda_{j}$ and eigenfunctions $\phi_{j}(\mathbf{x})$ obeying

$$
\int \mathrm{d} \mathbf{x}^{\prime} K\left(\mathbf{x}, \mathbf{x}^{\prime}\right) \phi_{j}\left(\mathbf{x}^{\prime}\right)=\lambda_{j} \phi_{j}(\mathbf{x})
$$

and the normalization

$$
\int \mathrm{d} \mathbf{x} \phi_{j}(\mathbf{x}) \phi_{j^{\prime}}\left(\mathbf{x}^{T}\right)=\delta_{j j^{\prime}}
$$

The above considerations show that in the limit of large $N$ the Laplace transform of the partition function, eq. (55), can again be expressed as

$$
g_{N}(t)=\left[\lambda_{0}(t)\right]^{N}
$$

where $\lambda_{0}$ is the largest eigenvalue of the transfer operator (56) which parametrically depends on $t$. Hence the form of the partition function and the saddle point equation will also be the same as in the last section, see eqs. (43) and (44).

In the following it will be useful to introduce the functions $\psi_{j}(t ; \mathbf{x})$ by writing $\phi_{j}(t ; \mathbf{x})$ in the form (again we indicate the $t$-dependence explicitly)

$$
\phi_{j}(t ; \mathbf{x})=\psi_{j}(t ; \mathbf{x}) \sqrt{R(t ; \mathbf{x})}
$$

where

$$
\begin{array}{r}
R(t ; \mathbf{x})=\exp \left(-t \sum_{j=1}^{k-1} x_{j}\right) \times \\
\prod_{j=0}^{k-2} \prod_{i=1}^{k-j-1} f\left(x_{i}+\ldots+x_{i+j}\right) .
\end{array}
$$

From eq. (59) we obtain the equation 


$$
\begin{gathered}
\int_{0}^{\infty} \mathrm{d} \xi_{k} e^{-t \xi_{k}} f\left(\xi_{k}\right) f\left(\xi_{k}+\xi_{k-1}\right) \ldots f\left(\xi_{k}+\ldots+\xi_{1}\right) \times \\
\psi_{j}\left(t ; \xi_{2}, \ldots, \xi_{k}\right)=\lambda_{j}(t) \psi_{j}\left(t ; \xi_{1}, \ldots, \xi_{k-1}\right)
\end{gathered}
$$

which in conjunction with the normalization condition

$$
\int_{0}^{\infty} \mathrm{d} \mathbf{x} R(t ; \mathbf{x}) \psi_{j}(t ; \mathbf{x}) \psi_{j^{\prime}}\left(t ; \mathbf{x}^{T}\right)=\delta_{j j^{\prime}}
$$

determines $\psi_{j}(t ; \mathbf{x})$ and $\lambda_{j}(t)$.

For the calculation of the joint probability distribution of $n$ consecutive spacings, eq. (18), we follow the same steps as in the last section, except that we have to distinguish two cases, namely $n<k-1$ and $n \geq k-1$. In the former case,

$$
\begin{aligned}
& p\left(\xi_{1}, \ldots, \xi_{n}\right)=\int_{0}^{\infty} \mathrm{d} \xi_{n+1} \ldots \int_{0}^{\infty} \mathrm{d} \xi_{k-1} \\
& \phi_{0}\left(c ; \xi_{1}, \ldots, \xi_{k-1}\right) \phi_{0}\left(c ; \xi_{k-1}, \ldots, \xi_{1}\right),
\end{aligned}
$$

where $c$ is determined from the saddle point equation (44), while in the latter case,

$$
\begin{gathered}
p\left(\xi_{1}, \ldots, \xi_{n}\right)=\lambda_{0}^{-n+k-1} \psi_{0}\left(c ; \xi_{n}, \ldots, \xi_{n-k+2}\right) e^{-c \sum_{i=1}^{n} \xi_{i}} \\
\left(\prod_{j=0}^{k-1} \prod_{i=1}^{n-j} f\left(\xi_{i}, \ldots, \xi_{i+j}\right)\right) \psi_{0}\left(c ; \xi_{1}, \ldots, \xi_{k-1}\right) .
\end{gathered}
$$

Eqs. (66) and (67) in conjunction with eq. (20) allow the calculation of the spacing distributions. For example, the nearest-neighbor spacing distribution becomes

$$
\begin{aligned}
P(s)= & \int_{0}^{\infty} \mathrm{d} \xi_{1} \ldots \int_{0}^{\infty} \mathrm{d} \xi_{k-1} \delta\left(s-\xi_{j} / \Delta\right) \\
& \phi_{0}\left(c ; \xi_{1}, \ldots, \xi_{k-1}\right) \phi_{0}\left(c ; \xi_{k-1}, \ldots, \xi_{1}\right),
\end{aligned}
$$

where $j$ may take any value from 1 to $\mathrm{k}-1$. In order to determine the asymptotic behavior of the number variance, we follow the same procedure as in the last section, i.e. we calculate the Laplace transform $g(n, t)$ of the $n$-th nearest-neighbor spacing distribution, sum this over $n$ to obtain the Laplace transform of the two-point correlation function, see eq. (19), and find its small- $t$ asymptotic, which determines the asymptotic behavior of the number variance, see eq. (24). For $n<k-2$ we get

$$
\begin{aligned}
& g(n, t)=\int_{0}^{\infty} \mathrm{d} \xi_{1} \ldots \int_{0}^{\infty} \mathrm{d} \xi_{n+1} e^{-\frac{t}{\Delta} \sum_{i=1}^{n+1} \xi_{i}} \int_{0}^{\infty} \mathrm{d} \xi_{n+2} \ldots \\
& \ldots \int_{0}^{\infty} \mathrm{d} \xi_{k-1} \phi_{0}\left(c ; \xi_{1}, \ldots, \xi_{k-1}\right) \phi_{0}\left(c ; \xi_{k-1}, \ldots, \xi_{1}\right),
\end{aligned}
$$


whose behavior for small $t$ is simply $g(n, t)=1+O(t)$ due to the normalization eq. (60). For $n \geq k-2$ we find

$$
\begin{gathered}
g(n, t)=\sum_{j}\left(\frac{\lambda_{j}(c+t / \Delta)}{\lambda_{0}(c)}\right)^{n-k+2}\left[\int_{0}^{\infty} \mathrm{d} \xi_{1} \ldots \int_{0}^{\infty} \mathrm{d} \xi_{k-1}\right. \\
\left.e^{-\frac{t}{2 \Delta} \sum_{i=1}^{k-1} \xi_{i}} \phi_{0}\left(c ; \xi_{1}, \ldots, \xi_{k-1}\right) \phi_{j}\left(c+\frac{t}{\Delta} ; \xi_{k-1}, \ldots, \xi_{1}\right)\right]^{2} .
\end{gathered}
$$

Summing over $n$ and expanding asymptotically for small $t$ we obtain

$$
\sum_{n=k-2}^{\infty} g(n, t)=\frac{1}{t}-\frac{1}{2 \Delta} \frac{\lambda_{0}^{\prime \prime}(c)}{\lambda_{0}^{\prime}(c)}-(k-1)+O(t),
$$

where we have used the saddle point equation (44) and $\int \mathrm{d} s s P(s)=1$ with $P(s)$ given by eq. (68). Since the $k-2$ remaining terms each contribute $1+O(t)$, we finally get

$$
g_{2}(t)=\frac{1}{t}-\frac{1}{2 \Delta} \frac{\lambda_{0}^{\prime \prime}(c)}{\lambda_{0}^{\prime}(c)}-1+O(t)
$$

Note that this expression depends on $k$ only by the largest eigenvalue of the corresponding transfer operator and it is identical with eq. (50).

At this point we again specialize to the random matrix interaction eq. (17) with integer $\beta$. The integral equation (64) which determines $\lambda_{j}(t)$ and $\psi_{j}(t ; \mathbf{x})$ then reads

$$
\begin{array}{r}
\int_{0}^{\infty} \mathrm{d} \xi_{k} \xi_{k}^{\beta}\left(\xi_{k}+\xi_{k-1}\right)^{\beta} \ldots\left(\xi_{k}+\ldots+\xi_{1}\right)^{\beta} e^{-t \xi_{k}} \times \\
\psi_{j}\left(t ; \xi_{2}, \ldots, \xi_{k}\right)=\lambda_{j}(t) \psi_{j}\left(t ; \xi_{1}, \ldots, \xi_{k-1}\right)
\end{array}
$$

The $t$-dependence of the eigenvalues and eigenfunctions factorizes and

$$
\begin{aligned}
\lambda_{j}(t) & =t^{-k \beta-1} \lambda_{j}(1), \\
\psi_{j}(t ; \mathbf{x}) & =t^{(k-1)(k \beta+2) / 4} \psi(1 ; t \mathbf{x}) .
\end{aligned}
$$

This permits us to determine $c$ from the saddle point equation (44), which yields

$$
c=\frac{k \beta+1}{\Delta}
$$

Using eqs. (23), (24), and (69) we then find the asymptotic behavior of the number variance,

$$
\Sigma^{2}(L) \sim \chi L, \quad \chi=\frac{1}{k \beta+1} \quad(L \gg 1) .
$$

In critical statistics this quantity is related with a certain (multi)fractal dimension [17]

$$
\chi=\frac{\eta}{2 d}
$$

where $d$ is the dimensionality of the system and $\eta=d-D_{2}$ and $D_{2}$ is one of the multifractional exponent defined through the behavior of the mean inverse participation ratio with the size of the system, $L$, 


$$
\left\langle\int d^{d} x\left|\psi_{n}(x)\right|^{4}\right\rangle \sim L^{-D_{2}}
$$

In our model geometrical interpretation of non-zero values of $\chi(73)$ remains unclear.

In order to find the spacing distributions explicitly we need to determine $\psi_{0}$ and $\lambda_{0}$ from eq. (70) and then use eq. (66) or (67) together with eq. (20). The solutions of eq. (70) have the form

$$
\begin{aligned}
\psi\left(t ; \xi_{1}, \ldots, \xi_{k-1}\right)= & \sum_{i_{1}=0}^{\beta} \sum_{i_{2}=0}^{3 \beta} \ldots \sum_{i_{k-1}=0}^{(k-1) k / 2} \\
& a_{i_{1} i_{2} \ldots i_{k-1}} \xi_{1}^{i_{1}} \xi_{2}^{i_{2}} \ldots \xi_{k-1}^{i_{k-1}} .
\end{aligned}
$$

The coefficients $a_{i_{1} i_{2} \ldots i_{k-1}}$ must be determined numerically by substituting eq. (76) into eq. (70). In tables [I, [I], and III the explicit form of $P(s)$ and $P(1, s)$ are shown for $k=1,2$, and 3 respectively, where in each case the distributions for $\beta=1,2$, and 4 are given.

These calculations become more and more tedious as $k$ or $\beta$ increase. However it is straightforward to find the large $s$ asymptotic of the spacing distributions,

$$
P(n, s) \sim e^{-\Lambda s}, \quad \Lambda=k \beta+1 \quad(s \gg 1) .
$$

Note that in our model the asymptotics of the spacing distributions and the number variance are related as follows,

$$
\Lambda=\frac{1}{\chi}
$$

For critical distribution of of the Anderson model at MIT point it is often assumed [18] that

$$
\Lambda=\frac{1}{2 \chi}
$$

To derive this relation it was assumed [18] that the probability to find $n$ levels in an interval which contains in average $L$ levels has the Gaussian form

$$
P_{n}(L) \sim \exp \left(-\frac{(n-L)^{2}}{2 \Sigma^{2}(L)}\right),
$$

where $\Sigma^{2}(L)$ as above is the number variance.

Therefore the probability that there is no levels inside this interval (i.e. the nearestneighbor distribution) is

$$
P_{0}(L) \sim \exp \left(-\frac{L^{2}}{2 \Sigma^{2}(L)}\right) .
$$

Assuming that, when $L \rightarrow \infty, \Sigma^{2}(L) \rightarrow \chi L$ one gets Eq. (79).

But it is clear that the assumption that $P_{n}(L)$ has the Gaussian form (80) even for small $n$ is an oversimplification and cannot be strictly valid in general. In our short-range plasma model $P_{n}(L)$ is like the one for the Poissonian process which gives Eq. (78). In the Gaudin 
model with $\beta=2$ there is no simple relation between $\Lambda$ and $\chi$ (see Eqs (10) and (11) (though for small $\chi$ one obtains Eq. (79)) .

This difference between the Gaudin model when $\chi \rightarrow 0$ and the relation (78) derived in our short range plasma model is probably related with the different truncation of the interaction between two levels. In the former model the distance between two levels is important while in the later one only the number of levels between the two given levels is taken into account.

Numerical calculations of the Anderson model at the MIT point and certain analytical arguments [25], [26] based on the kernel (13) are in the favor of the relation (79) at least in the limit of small $\chi$. The short range plasma model demonstrates that there exist (mathematical) models of intermediate statistics which do not obey this relation.

\section{SPECTRAL STATISTICS OF PSEUDOINTEGRABLE BILLIARDS}

Pseudointegrable systems, as introduced by Richens and Berry [19], are dynamical systems which are neither integrable nor chaotic. The difference between integrable and pseudointegrable systems is that for the former the phase space is foliated into 2-dimensional surfaces with genus $g=1$ (i.e. tori) while for the latter it is foliated into surfaces of higher genus. The simplest example of pseudointegrable systems is plane polygonal billiards whose angles are all rational multiples of $\pi$. For these models the genus of the corresponding surface is given by

$$
g=1+\frac{N}{2} \sum_{k} \frac{m_{k}-1}{n_{k}},
$$

where $m_{k} \pi / n_{k}$ are the interior vertex angles of the polygon, $N$ is the least common multiple of the integers $n_{k}$, and the sum is taken over all vertices of the polygon. The polygons with $g=1$ (e.g. rectangle, equilateral triangle) are integrable, whereas those with $g \geq 2$ are pseudointegrable. In Refs. [20], [21] the spectral statistics of a number of pseudointegrable polygonal billiards have been investigated numerically (and in the last reference even experimentally using a microcavity) and it was found that they display level repulsion but deviate from random matrix theory.

In [10] a number of different models had been considered numerically which clearly demonstrate the existence of intermediate statistics. In this Section we present a more detailed analysis of the data.

We consider the sequence of pseudointegrable billiards (with Dirichlet boundary conditions) in the shape of the right triangle with one angle equal $\pi / n$, where $n=5,7,8,9, \ldots, 30$ (the triangles with $n=3,4,6$ are integrable) and using the boundary integral method we have calculated the first 20000-30000 levels for all these triangles (for some triangles even up to 80000 levels).

Figure 2 shows the difference between the cumulative nearest-neighbor spacing distribution

$$
N(s)=\int_{0}^{s} \mathrm{~d} x P(x)
$$

(calculated from the levels 5000-20000) for all triangles and the Semi-Poisson prediction 


$$
N_{1}(s)=1-(2 s+1) \exp (-2 s)
$$

which is obtained from our model with $\beta=k=1$ (see Eq. (36)).

Roughly four close groups of curves are observed. For the first group, consisting of the curves corresponding to triangles with $n=5,8,10,12$, this difference is less than one percent and consequently the spectral statistics of these billiards is quite well described by the SemiPoisson model. The remaining three groups correspond to the triangles with $n=7,14,18$, with $n=9,16,20,24,30$, and the rest.

The first conclusion from this and many others figures is that spectral statistics of pseudointegrable systems is not universal and depends on the billiard angles. The grouping of the triangles which emerges from Fig. 2 does not agree with the classification of the billiards according to their geometrical genus $g$. In first approximation the spectral statistics of the above triangular billiards are reasonably well described by a quantity $q$ which we proposed to call 'arithmetical genus'

$$
q(n)=\left\{\begin{array}{cc}
g(n), & n \text { odd } \\
\phi(n) / 2, & n \text { even }
\end{array} .\right.
$$

Hence $q$ is equal to the 'geometrical genus' $g$ for $n$ odd but for even $n$ it is given by half the Euler function $\phi(n)$ (equal to the number of integers not exceeding and relatively prime to $n$ ). The first group now correspond to the triangles with $q=2$, the second to $q=3$, the third to $q=4$, and the last to $q>4$. It seems that for $q>4$ the spacing distribution does not change noticeably any more, but the resulting distribution may differs from the WignerDyson spacing distribution. We stress that this classification is only an approximative one and serves mostly for the crude arrangement of the spectral statistics of different triangles.

In Figure 3 we present the evolution of the cumulative nearest-neighbor distribution for the triangular billiard with $n=12$ with increasing energy. It is clearly seen that for higher energy the spacing distribution moves closer to the Semi-Poisson result (though a limiting distribution may deviates from the Semi-Poisson one).

In Figure 1 the difference between the cumulative next-to-nearest distribution for the same triangle and the Semi-Poisson prediction (see Table $\mathbb{I}$ ) is plotted. Once more we observed that this distribution is close to the Semi-Poisson result and that, with increasing energy, the agreement is better.

The main conclusion of this Section is that for certain pseudointegrable systems the short-range spectral statistics is very close to the Semi-Poisson statistics (though theoretical explication of this fact is not yet clear).

\section{SUMMARY AND DISCUSSION}

In order to model intermediate spectral statistics, we considered a one-dimensional gas of energy levels interacting via a logarithmic pair potential, whose action is restricted to a small number of nearest neighbors. As shown in section II, its correlation functions can be calculated analytically, so the model deserves interest as a simple reference point for comparisons with numerics. In section [II] we performed a comparison with the spectral statistics

of a number of pseudointegrable billiards and demonstrated that the plasma model with 
nearest-neighbor interaction (the Semi-Poisson model) gives an excellent phenomenological description of the short-range spectral observables of certain pseudointegrable systems. Unfortunately a full theoretical understanding of the spectral statistics of pseudointegrable systems is still lacking.

Of course there are many ways to interpolate between Wigner-Dyson and Poisson statistics. For the spacing distribution a well-known interpolation is the Brody distribution [22], which shows fractional level repulsion. Since for intermediate spectral statistics $P(s) \sim s^{\beta}$ for small spacings, the Brody distribution cannot give an adequate description. A very natural way to construct interpolating ensembles is to take a weighted average between the ensemble of diagonal random matrices and one of the standard random matrix ensembles, e.g. for $\beta=1$

$$
H=H_{\text {Poisson }}+\lambda H_{\mathrm{GOE}}
$$

(see e.g. ref. 23]). However, as we checked numerically, for no value of $\lambda$ the resulting distribution is close to the distribution (8), which we have found to be an excellent fit to the spacing distribution of the pseudointegrable triangles with $q=2$ and also to that of several other systems [10]. Hence this interpolation do not seem to be suitable for the description of intermediate statistics.

All data suggest that a necessary requirement of theoretical description of intermediate statistics is the screening of two-body potential. Its precise form is not yet known (but see [24]- 26] ) and may depend on the problem considered.

\section{ACKNOWLEDGMENTS}

The authors is greatly indebted to A. Pandey who had investigated the short-range plasma model in the framework of band random matrices and whose unpublished notes were useful to check our calculations.

It is a pleasure to thank O. Bohigas, D. Delande, A. Mirlin and G. Montambaux for useful discussions. UG acknowledges a HSP2-fellowship of the 'Deutscher Akademischer Austauschdienst', and thanks the IPN Orsay for kind hospitality during an extended stay. 


\section{REFERENCES}

[1] C. E. Porter, Statistical theories of spectra: fluctuations (Academic Press, New York, 1965).

[2] M. L. Mehta, Random matrices, 2nd ed. (Academic Press, New York, 1991).

[3] O. Bohigas in Chaos and Quantum Physics, Proceedings of the Les Houges Summer School of Theoretical Physics, 1989, eds. M.-J. Giannoni, A. Voros, and J. Zinn-Justin (Elsevier, New York 1991).

[4] T. Guhr, A. Müller-Groeling, and H. A. Weidenmüller, Phys. Rep. 299, 190 (1998).

[5] E. Bogomolny, O. Bohigas, and M.P. Pato, Phys. Rev. E 55, 6707 (1997).

[6] B. I. Shklovskii et al., Phys. Rev. B 47, 11487 (1993).

[7] Y. V. Fyodorov and A. D. Mirlin, Phys. Rev. B 55, R16001 (1997).

[8] M.V. Berry and M. Tabor, Proc. R. Soc. London A 356, 375 (1977).

[9] O. Bohigas, M.-J. Giannoni, and C. Schmit, Phys. Rev. Lett. 52, 1 (1984); ibid J. Physique Lett. 45. L1015 (1984).

[10] E. Bogomolny, U. Gerland, and C. Schmit, Phys. Rev. E 59, R1315 (1999).

[11] M. Gaudin, Nucl. Phys. 85, 545 (1966).

[12] P. J. Forrester, Phys. Lett. A 173, 355 (1993).

[13] F. Gürsey, Proc. Cambridge Phil. Soc. 46, 182 (1950).

[14] L. van Hove, Physica 16, 137 (1950).

[15] E. H. Lieb and D. C. Mattis, Mathematical Physics in One Dimension (Academic Press, New York, 1966).

[16] H. Hernández-Saldaña, J. Flores, and T. H. Seligman, Phys. Rev. E 60, 449 (1999).

[17] J.T. Chalker, V.E. Kravtsov, and I.V. Lerner, JETP Lett., 64, 386 (1996).

[18] B. Al'tshuler, I. Kh. Zharekeshev, S.A. Kotochigova, and B.I. Shklovskii, Sov. Phys. JETP, 67, 625 (1988).

[19] P. J. Richens and M. V. Berry, Physica D 2, 495 (1981).

[20] A. Shudo and Y. Shimizu, Phys. Rev. E 47, 54 (1993).

[21] A. Shudo, Y. Shimizu, P. Šeba, J. Stein, H. J. Stöckmann, and K. Zyczkowski, Phys. Rev. E 49, 3748 (1994).

[22] T. A. Brody, Lett. Nuov. Cim. 7, 482 (1973).

[23] T. Guhr and H. A. Weidenmüller, Ann. Phys. 193, 472 (1989).

[24] V.E. Kravtsov and I.V. Lerner, J. Phys. A 28, 3623 (1995).

[25] S.M. Nishigaki, Phys. Rev. E 59, 2853 (1999).

[26] V.E. Kravtsov, A.M. Tsvelik, cont-mat/0002120 (2000). 


\section{FIGURES}

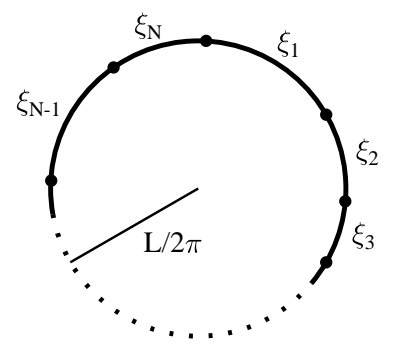

FIG. 1. $N$ particles on a circle of circumference $L$. The positive spacings between consecutive particles measured along the circle are denoted by $\xi_{j}$ with $j=1 \ldots N$.

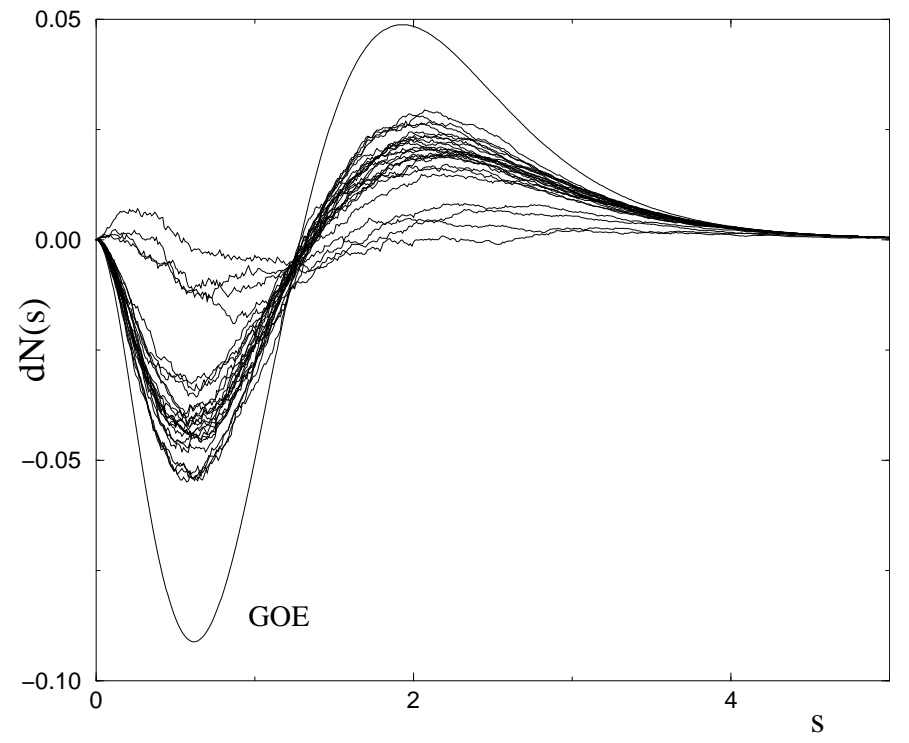

FIG. 2. Difference between the cumulative spacing distribution for the pseudointegrable rational right triangles with $n \leq 30$ (calculated from the levels 5000-20000) and the Semi-Poisson curve. See explanation in the text. 


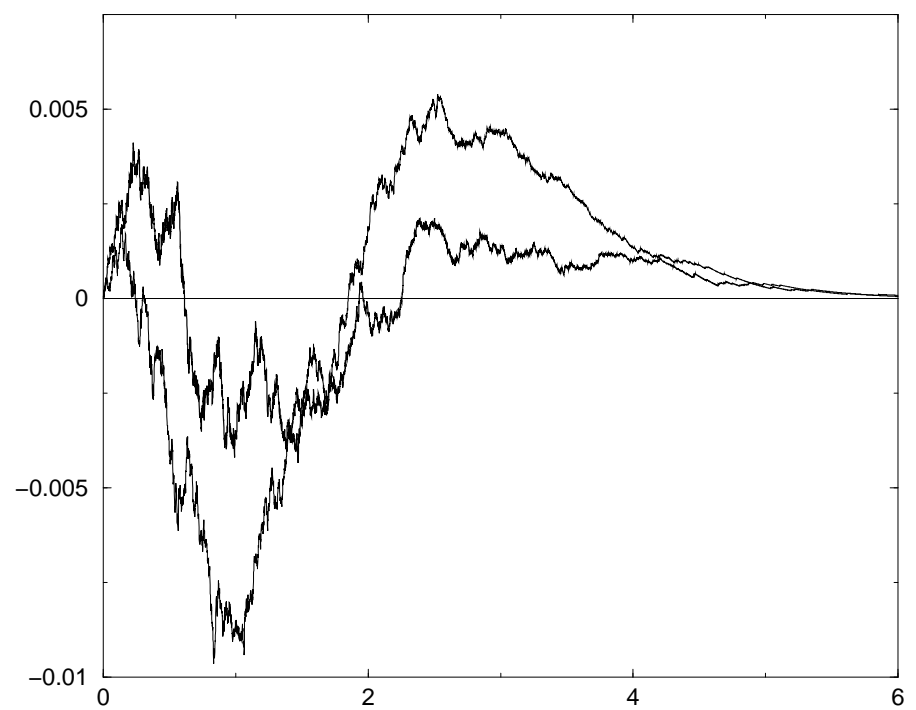

FIG. 3. The same as in Fig. 2 but for $n=12$. The §hin line corresponds to 1-34000 levels and the thick one includes 34001-68000 levels.

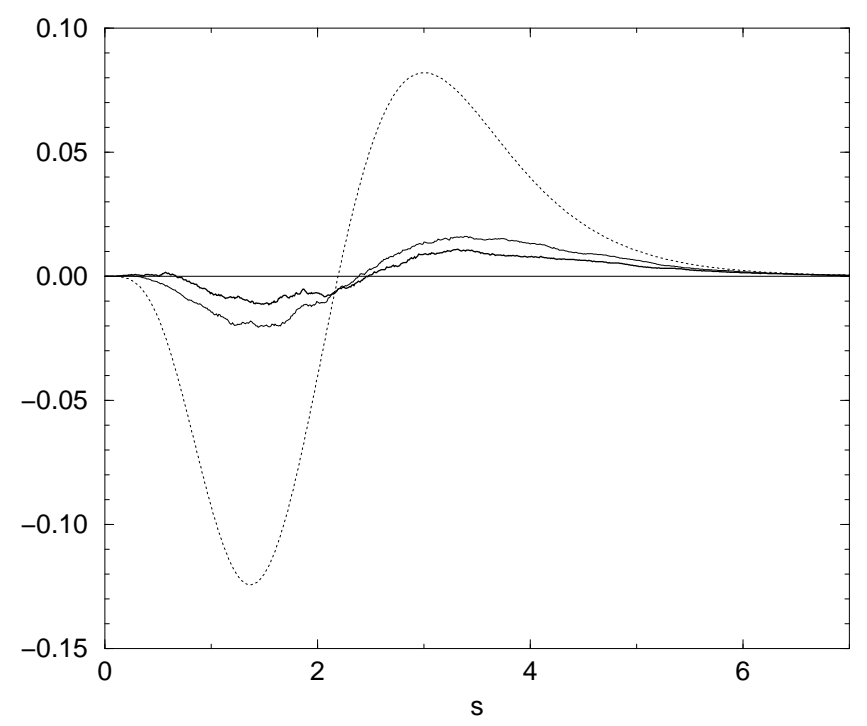

FIG. 4. The difference between the cumulative next-to-nearest spacing distribution for $n=12$ and the Semi-Poisson prediction. Two curves are the same as in Fig. 3. The dotted line corresponds to GOE 


\section{TABLES}

TABLE I. The spacing distributions $P(s)$ and $P(1, s)$ for the one-dimensional gas model with nearest-neighbor interaction.

\begin{tabular}{lccc}
\hline \hline$(k=1)$ & $\beta=1$ & $\beta=2$ & $\beta=4$ \\
\hline$P(s)$ & $4 s e^{-2 s}$ & $27 / 2 s^{2} e^{-3 s}$ & $3125 / 24 s^{4} e^{-5 s}$ \\
$P(1, s)$ & $8 / 3 s^{3} e^{-2 s}$ & $243 / 40 s^{5} e^{-3 s}$ & $1953125 / 72576 s^{9} e^{-5 s}$ \\
\hline \hline
\end{tabular}

TABLE II. Same as Tab. 目, but with nearest- and next-to-nearest-neighbor interaction.

\begin{tabular}{lccc}
\hline \hline$(k=2)$ & $\beta=1$ & $\beta=2$ & $\beta=4$ \\
\hline & $c_{0}=2.4773$ & $c_{0}=6.2603$ & $c_{0}=35.6018, c_{1}=252.5852$ \\
$P(s)=s^{\beta} e^{-(2 \beta+1) s} \sum_{j} c_{j} s^{j}$ & $c_{1}=6.0681$ & $c_{1}=24.9958$ & $c_{2}=826.3343, c_{3}=1630.9839$ \\
& $c_{2}=3.7159$ & $c_{2}=41.1181$ & $c_{4}=2127.3725, c_{5}=1880.5911$ \\
& & $c_{3}=32.2768$ & $c_{6}=1103.6323, c_{7}=395.1819$ \\
& & $c_{4}=10.4386$ & $c_{8}=66.6078$ \\
& $d_{0}=2.5054$ & $d_{0}=5.9771$ & $d_{0}=29.4495, d_{1}=104.4681$ \\
& $d_{1}=3.068$ & $d_{1}=11.9326$ & $d_{2}=169.5742, d_{3}=164.1739$ \\
& $d_{2}=0.7516$ & $d_{2}=9.5151$ & $d_{4}=103.3668, d_{5}=43.2920$ \\
& & $d_{3}=3.3018$ & $d_{6}=11.9204, d_{7}=2.0002$ \\
& & $d_{4}=0.4746$ & $d_{8}=0.1586$ \\
\hline \hline
\end{tabular}


TABLE III. Same as Tab. [, but with three interacting neighbors.

\begin{tabular}{|c|c|c|c|}
\hline$(k=3)$ & $\beta=1$ & $\beta=2$ & $\beta=4$ \\
\hline \multirow{11}{*}{$P(s)=s^{\beta} e^{-(3 \beta+1) s} \sum_{j} c_{j} s^{j}$} & $c_{0}=2.1342$ & $c_{0}=4.9711$ & $c_{0}=24.0711, c_{1}=282.9984$ \\
\hline & $c_{1}=7.8196$ & $c_{1}=31.6156$ & $c_{2}=1615.27, c_{3}=5952.78$ \\
\hline & $c_{2}=11.6945$ & $c_{2}=93.9581$ & $c_{4}=15890.11, c_{5}=32666.46$ \\
\hline & $c_{3}=9.0743$ & $c_{3}=171.4838$ & $c_{6}=53678.64, c_{7}=72222.20$ \\
\hline & $c_{4}=3.6855$ & $c_{4}=212.8904$ & $c_{8}=80836.91, c_{9}=76044.48$ \\
\hline & $c_{5}=0.6259$ & $c_{5}=188.1012$ & $c_{10}=60480.73, c_{11}=40759.87$ \\
\hline & & $c_{6}=120.0046$ & $c_{12}=23247.21, c_{13}=11165.36$ \\
\hline & & $c_{7}=54.6739$ & $c_{14}=4473.73, c_{15}=1472.98$ \\
\hline & & $c_{8}=17.0418$ & $c_{16}=389.3105, c_{17}=79.6241$ \\
\hline & & $c_{9}=3.2829$ & $c_{18}=11.8577, c_{19}=1.1466$ \\
\hline & & $c_{10}=0.2968$ & $c_{20}=0.0541$ \\
\hline \multirow{13}{*}{$P(1, s)=s^{3 \beta+1} e^{-(3 \beta+1) s} \sum_{j} d_{j} s^{j}$} & $d_{0}=1.1340$ & $d_{0}=1.6110$ & $d_{0}=2.9196, d_{1}=29.5956$ \\
\hline & $d_{1}=3.6334$ & $d_{1}=8.8782$ & $d_{2}=146.1401, d_{3}=467.9231$ \\
\hline & $d_{2}=5.0189$ & $d_{2}=23.0847$ & $d_{4}=1090.9150, d_{5}=1971.2442$ \\
\hline & $d_{3}=3.7112$ & $d_{3}=37.4284$ & $d_{6}=2868.9524, d_{7}=3449.8348$ \\
\hline & $d_{4}=1.5037$ & $d_{4}=42.0839$ & $d_{8}=3488.0347, d_{9}=3001.6809$ \\
\hline & $d_{5}=0.3100$ & $d_{5}=34.4593$ & $d_{10}=2216.98, d_{11}=1412.97$ \\
\hline & $d_{6}=0.0259$ & $d_{6}=20.9804$ & $d_{12}=779.3162, d_{13}=372.3224$ \\
\hline & & $d_{7}=9.5327$ & $d_{14}=153.8623, d_{15}=54.8050$ \\
\hline & & $d_{8}=3.2010$ & $d_{16}=16.7526, d_{17}=4.3271$ \\
\hline & & $d_{9}=0.7747$ & $d_{18}=0.94224, d_{19}=0.1687$ \\
\hline & & $d_{10}=0.1284$ & $d_{20}=0.0241, d_{21}=2.76 * 10^{-3}$ \\
\hline & & $d_{11}=0.0131$ & $d_{22}=2.5 * 10^{-4}, d_{23}=1.9 * 10^{-4}$ \\
\hline & & $d_{12}=6$ & $d_{24}$ \\
\hline
\end{tabular}

